\title{
Comparative Study on CBIR based by Color Histogram, Gabor and Wavelet Transform
}

\author{
D. Ashok Kumar \\ Department of Computer Science \\ V.S.S Government Arts College \\ Pulankurichi - 630405
}

\author{
J. Esther \\ Department of Computer Science \\ Mano College \\ Govindaperi- 627414
}

\begin{abstract}
Content-Based Image Retrieval (CBIR), also known as query by image content (QBIC) Content-based image retrieval (CBIR) system helps users to retrieve relevant images based on their contents. Content Based Image Retrieval (CBIR) technologies provide a method to find images in large databases by using unique descriptors from a trained image. The image descriptors include texture, color, intensity and shape of the object inside an image. In this paper, we compare the several feature extraction techniques viz., Gabor, Wavelet and Histogram over color and texture features applied in a novel way for color image and used for CBIR. The experiments which show that the Gabor filters advice promising results are reported.
\end{abstract}

\section{General Terms}

Image Processing, CBIR.

\section{Keywords}

CBIR, Histogram, Gabor and Wavelet. Transform.

\section{INTRODUCTION}

Impression is more by an image rather than thousands of words as stipulated by the statement "A picture is worth a thousand words". Nowadays, because of the Internet and the image sensor technology improvement, it is easy to access to digital images and the large amount of multimedia data are generated and transmitted in digital format. When search for an image from the web the results cannot meet their satisfaction. Even with the Google image search, the user's requirement still cannot be completely satisfied. Since searching of images are based upon associated metadata such as keywords, text, etc. The aim of CBIR is to avoid the use of textual descriptions. So in CBIR, retrieving of image based on similarities in their contents like textures, colors, shapes etc. are lower level features of image. The term CBIR describes the process of retrieving desired images from the large collection of database on the basis of features that can be automatically extracted from the images themselves.

In CBIR each image stored in the database, has its features extracted and compared to the features of the query image. It involves two processes viz Feature extraction and feature matching process.

In the first process it will extract the image features to a distinguishable extent. In this paper Color Histogram for color feature; Gabor and wavelet Transform for texture feature are used and extract their features from the image. In the second process it involves matching these features to yield a result that is visually similar. In this paper three types of Distance Metric Measures like Euclidean Distance, Chi-Square Distance and Weighted Euclidean Distance for feature matching process are used. The basic idea behind CBIR is when building an image database, feature vectors from images like color, texture are to be extracted and storing the vectors in another database for future use.

For the given a query image its feature vectors are computed. If the distance between feature vectors of the query image and images in the database is small enough, the corresponding image in the database is to be considered as a match to the query. The search is usually based on similarity rather than on exact match and the retrieval results are then ranked accordingly to a similarity index. The block diagram of basic CBIR system is shown in Figure 1.

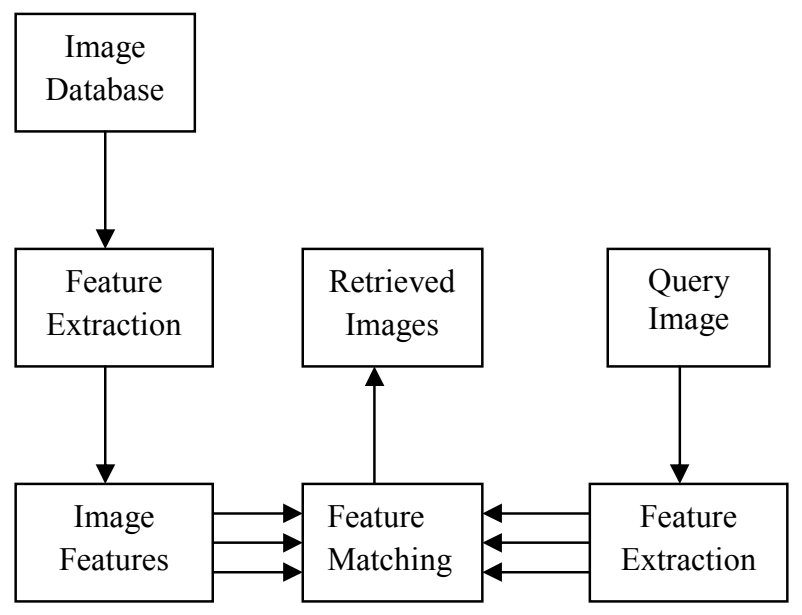

Figure 1. Block diagram of CBIR

For example a distance of 0 signifies an exact match with the query. In this paper, the detailed comparison of different feature extraction techniques are discussed and experimented on group of images. The motivation is to get the best technique to be used in further image retrieval application. This paper is organized as follows. The next section briefly describes the review of literature, Visual Feature Extraction methods are discussed in Section 3, Feature Matching Process are discussed in 4, Result and Discussions are in Section 5, Finally conclusion of the paper and presenting future research directions are in Section 6. 


\section{REVIEW OF LITERATURE}

Content-based image retrieval, also known as query by image content and content-based visual information retrieval is the application of computer vision to the image retrieval problem, that is, the problem of searching for digital images in large databases. In the first generation, text annotations are used to retrieve the image accordingly [1]. To overcome the difficulties encountered by a text-based image retrieval system, content-based image retrieval was proposed in the early 1990s [3, 4].Some successful applications of CBIR include:

- Texture segmentation and texture feature extraction (Fogel and Sagi, 1989; Jain and Farrokhnia, 1991; Turner, 1990),

- Fingerprints identification (Hammamoto, 1999),

- Face (Liu and Wechsler, 2001; Wiskott et al., 1997)

- Iris recognition (Daugman, 1998),

- Edge detection (Mehrotra et al., 1992; Su and Wang, 2003),

- Directional image enhancement, image compression (Daugman, 1988),

- Hierarchical image representation and recognition (Jain et al., 1997; Lee, 1996).

CBIR concept is used in many different fields, in Biomedicine fields such that X-ray, CT, medical diagnosis and security filtering, art galleries, museums, personal album etc.

A number of previous works have been done addressing different feature extraction techniques of the image elements for image retrieval. In 2008, Wan Siti Halimatul Munirah Wan Ahmad and Mohammad Faizal Ahmad Fauzi [8] have proposed feature extraction techniques in CBIR using CT Brain image (gray scale image). In this paper trials of implementing it in color images with feature extraction technique of Color Histogram (Color feature), Gabor Transform (texture feature) and Wavelet Transform with (texture feature) are adhered. Distance Metric Measures like Euclidean Distance, Chi-Square Distance, and Weighted Euclidean Distance for retrieving the image from the image dataset are used.

\section{VISUAL FEATURE EXTRACTION METHODS}

\subsection{Binning Method (Color Histogram):}

Color represents one of the most widely used visual features in CBIR systems. Each pixel of a image is associated to a specific histogram bin only on the basis of its own color, and color similarity across different bins or color dissimilarity in the same bins are not taken into account. Here three components like red, green, blue in RGB space are used. For the color histogram, the distribution of the number of pixels for each quantized bin can be defined for each component. Quantization in terms of color histograms refers to the process of reducing the number of bins by taking colors that are very similar to each other and putting them in the same bin. By default the maximum number of bins one can obtain using the color histogram function in MatLab is 256. The comparison between images (query image and image in database) is accomplished through the use of some distance metric which determines the distance or similarity between the two histograms.
Initially, the color features for all images and also for the query image are extracted. The Color histogram of image $\mathrm{I}$ is defined as, for a color $\mathrm{C}_{\mathrm{i}}, \mathrm{HC}_{\mathrm{i}}$ (I) represents the number of pixels of color $\mathrm{C}_{\mathrm{i}}$ in image I. for color histogram [9].

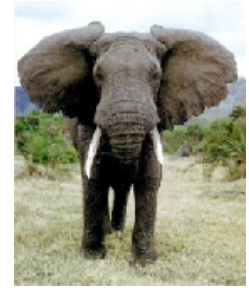

Figure 2(a ) Elephant as a sample query image

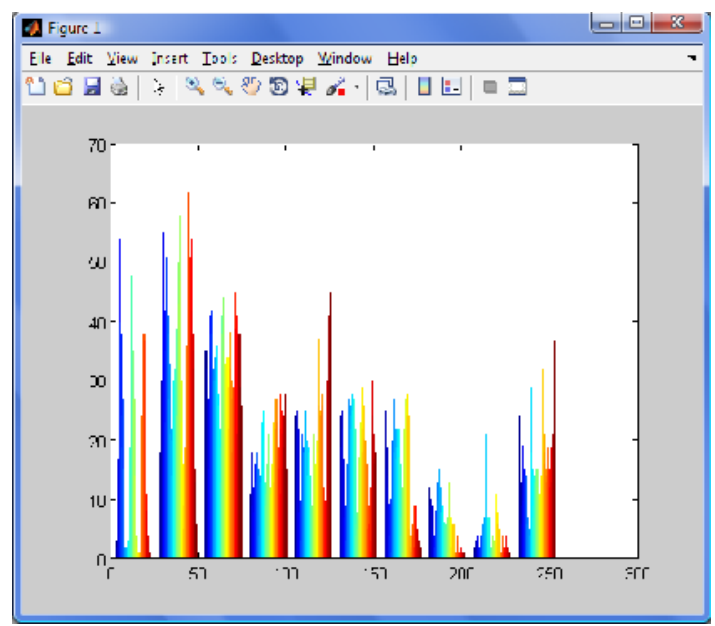

Figure 2(b). Histogram of Elephant image

The sample of query image and its histogram is shown in Figure 2(a) and Figure 2(b).

\subsection{Gabor Transform (Texture)}

Gabor is a technique that extracts information from an image. It's a multi-scale, multi-resolution filter. The two-dimensional Gabor filter can be represented as a complex sinusoidal signal modulated by Gaussian function as $\psi(x, y ; \sigma, \lambda, \theta k)$ [2] and

$\psi\left(x, y, \sigma, \lambda, \theta_{k}\right)=g(x, y, \sigma) \exp \left(\frac{2 \pi x \theta k}{\lambda} i\right)--(1)$ it can be formulated as follows: where

$$
g(x, y, \sigma)=\exp \left(-\frac{x^{2} \theta k+\gamma^{2} y^{2} \theta k}{2 \sigma^{2}}\right)---(2)
$$

is a Gaussian function, where

$$
\begin{aligned}
& x \theta_{k}=x \cos \left(\theta_{k}\right)+y \sin \left(\theta_{k}\right)----(3) \\
& y \theta_{k}=-x \sin \left(\theta_{k}\right)+y \sin \left(\theta_{k}\right)---(4)
\end{aligned}
$$

From (1) \& (2) $\sigma$ is the standard deviation of the Gaussian function with $\mathrm{x}, \mathrm{y}$ dimensions and $\lambda$ and $\theta_{k}$ are wavelength 
and orientation. The parameter $\gamma$ is usually equal to 0.5 . Gabor Filter with 4 scales and 5 orientations are design. Substitute the angle $\theta_{k}$ by $15,45,75,135$ and 180 respectively and the wavelengths are $60,80,120$ and 130 in equation (3) \& (4). After applying the Gabor Filter, the texture features of images are extracted by using mean function. For retrieving the images from the dataset, the Distance Metric Measures for every images are calculated. The minimum distance value signifies an exact match with the query. Gabor filters at different scales and spatial frequencies are shown in Figure 3. The top row shows asymmetric (or odd) filters, bottom row the symmetric (or even) filters.

$$
\begin{aligned}
& \text { symmetric: } \cos \left(k_{x} x+k_{y} y\right) \exp -\left\{\frac{x^{2}+y^{2}}{2 \sigma^{2}}\right\} \\
& \text { asymmetric: } \sin \left(k_{x} x+k_{y} y\right) \exp -\left\{\frac{x^{2}+y^{2}}{2 \sigma^{2}}\right\}
\end{aligned}
$$

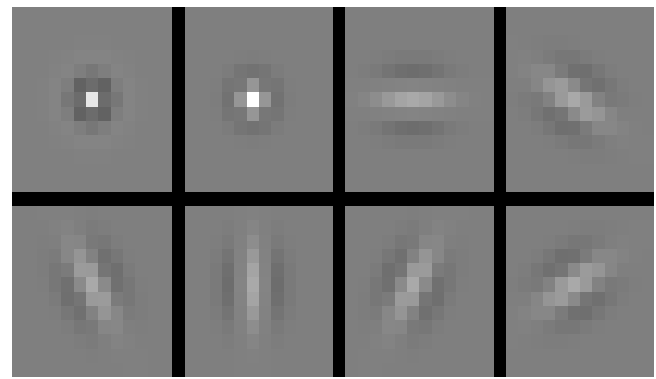

Figure 3. Gabor Filters with different frequency and wavelength

Gabor transform has selectivity for orientation, spectral bandwidth and spatial extent. The following Figure 4(a) shows that the input query image, and for that image by using the Gabor filter with different wavelength and orientation is shown in Figure 4(b).

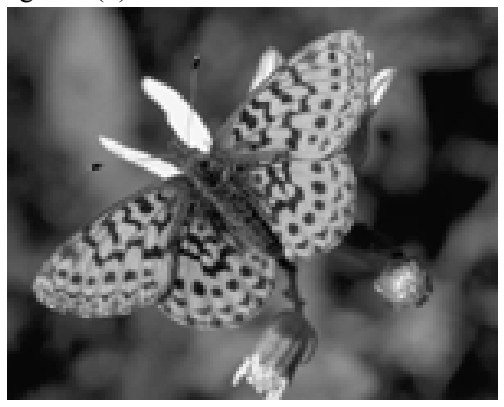

Figure 4(a) Example of input image
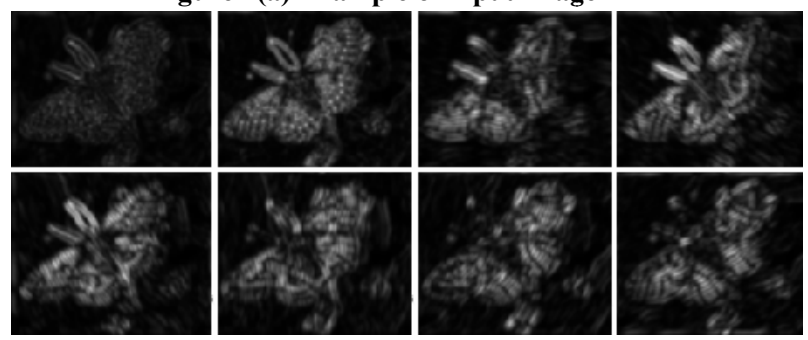

Figure 4(b) Example of different wavelength and orientations

\subsection{Wavelet Transform (Texture)}

Wavelet is the multi-resolution analysis of an image and also it is proved that having the signal of both space and frequency domain [5]. An image can be taken as a kind of two dimension signal; the wavelet coefficient can describe the image high frequency information and then can be taken as the image texture feature. Textures can be modeled as quasiperiodic patterns with spatial/frequency representation. For texture extraction, Wavelet decomposition of image regions is used. By applying Wavelet on the color image four sub images will be produced which is: A low resolution copy of original image, and three-band passed filters in specific directions: horizontal, vertical and diagonal respectively. These sub images contain information about texture characteristics. To have a numerical measure of texture, mean and variations of these images will be calculated.

The simplest orthogonal filter bank is Haar filter bank [2]. It applies two channel filter banks namely, from (5) low pass filter and from (6) high pass filter. The low pass filter is:

$h_{0}[n]=\left\{\begin{array}{l}\frac{1}{\sqrt{2}}, n=0,-1 \\ 0, \text { otherwise }\end{array}\right\}----(5)$

The high pass filter identifies the texture of the image. The low pass filter identifies the structure of the image.(i.e.) edges, curves etc. The high pass filter is:

$$
h_{1}[n]=\left\{\begin{array}{l}
\frac{1}{\sqrt{2}}, n=0 \\
-\frac{1}{\sqrt{2}}, n=-1 \\
0, \text { otherwise }
\end{array}\right\}---(6)
$$

Given an $\mathrm{N} \times \mathrm{N}$ image $\mathrm{F}$, the Haar transform will be computed as: $\mathrm{T}=$ HFHT, Where $\mathrm{H}$ contains the Haar basis functions. The matrix $\mathrm{H}$ will be defined with the structure:

$$
H_{N}=\left[\begin{array}{cccc}
h_{0}(0) & h_{0}\left(\frac{1}{N}\right) & \ldots . . & h_{0}\left(\frac{N-1}{N}\right) \\
h_{1}(0) & h_{1}\left(\frac{1}{N}\right) & \ldots . & h_{1}\left(\frac{N-1}{N}\right) \\
\ldots \ldots & \ldots \ldots . . & \ldots . . & \ldots \ldots . \\
h_{N-1}(0) & h_{N-1}\left(\frac{1}{N}\right) & \ldots \ldots & h_{N-1}\left(\frac{N-1}{N}\right)
\end{array}\right]
$$


$H_{0}(Z)=\frac{1}{\sqrt{2}}$ For all $Z$, this fixes the first row.

2 X 2 Haar transformation matrix is defined by:

$H_{2}=\frac{1}{\sqrt{2}}\left[\begin{array}{cc}1 & 1 \\ 1 & -1\end{array}\right]$

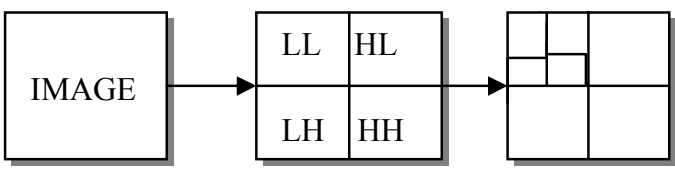

1 Level 2 Level

Figure 5. Hierarchical Subband Technique

The discrete wavelet transform is a hierarchical subband technique and it is shown in Figure 5.The subbands are created by applying decomposition on original image. To start the decomposition, the image is filtered in both the horizontal and vertical directions using separable filters [7]. This creates four subbands as follows:

- Subband LL1 represents the horizontal and vertical low frequency components of the image.

- Subband HH1 represents the horizontal and vertical high frequency components of the image.

- Subband LH1 represents the horizontal low and vertical high frequency components.

- Subband HL1 represents the horizontal high and vertical low frequency components.

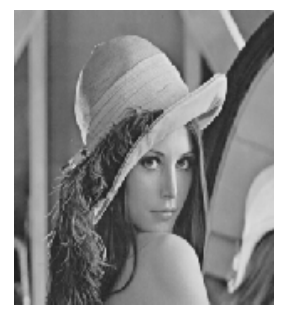

Figure 6(a) Sampl Image

The creation of subband technique with sample image is shown in Figure 6(a) and Figure 6(b) shows the two level decomposition of images and the Figure 6(c) shows the decomposition maps.

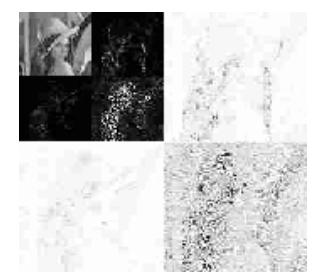

Figure 6(b) Two level Decomposition
To obtain the next level in the decomposition the two filters are again applied, but only to the LL1 subband. Again four subbands labeled LL2, HH2, LH2, and HL2 with representations similar to the first level subbands are obtained.

\begin{tabular}{|c|c|c|}
\hline LL2 & HL2 & \multirow{2}{*}{ HL1 } \\
\cline { 1 - 2 } LH2 & HH2 & \\
\hline LH1 & HH1 \\
& \\
\hline
\end{tabular}

Figure 6( c) Decomposition Map

\section{FEATURE MATCHING PROCESS}

To retrieve the similarity images from the large image dataset, three types of Distance Metric Measures like Euclidean Distance, Chi-Square Distance and Weighted Euclidean Distance are used.

\subsection{Euclidean Distance:}

The formula of Euclidean distance is

$$
\left.D=\sqrt{\sum\left(x_{i}-y_{i}\right.}\right)^{2}---(7) .
$$

The minimum distance value signifies an exact match with the query.

\subsection{Chi-Square Distance:}

The formula is $D^{2}=\left(\frac{\left(x_{i}-y_{i}\right)^{2}}{y_{i}}\right)---(8)$ where $x_{i}$ are the training image values. $y_{i}$ is the query image values. The minimum distance value signifies an exact match with the query.

\subsection{Weighted Euclidean Distance:}

$$
D=\sqrt{\sum W\left(x_{i}-y_{i}\right)^{2}}
$$

The minimum distance value signifies an exact match with the query. Here for the value of weight, denoted by $\mathrm{W}$ is assigned by 2 . [7].

\section{RESULTS \& DISCUSSION}

The experiments are conducted using Matlab 7.3 on an Intel Pentium-D $2.0 \mathrm{GHz}$ processor with $2 \mathrm{~GB}$ memory.In this experiment 800 images, representing 7 different objects like group of animals, flowers, sceneries, etc taken in various views are used. To perform the experimentations, the database by applying some image processing algorithms for extracting some low level characteristics is pre-processed. The data flow diagram is shown in Figure 7. 


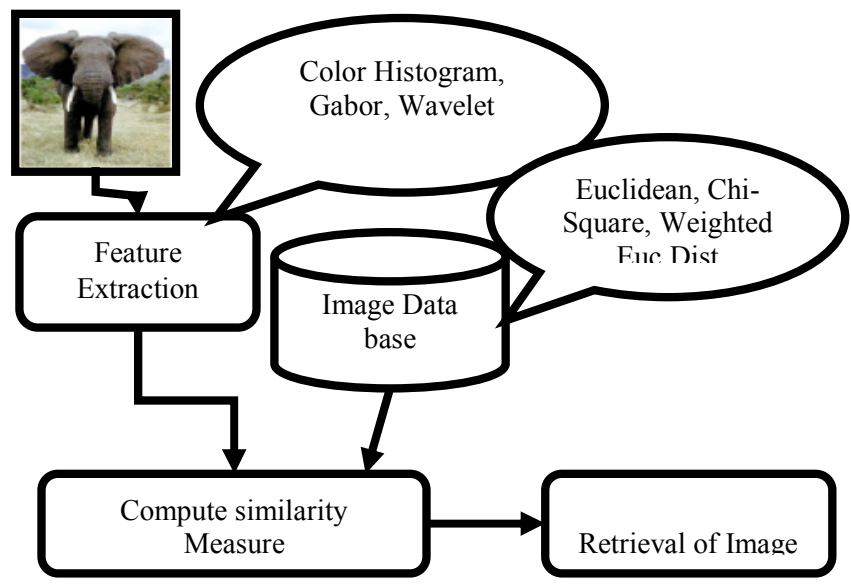

Figure 7 Data Flow diagram of CBIR

The retrieval of image consists of two stages, namely the offline feature extraction stage, and the online retrieval stage. During the offline stage, the two feature extraction techniques are applied to all color images in the database. The flow of off line process is shown in Figure 8.

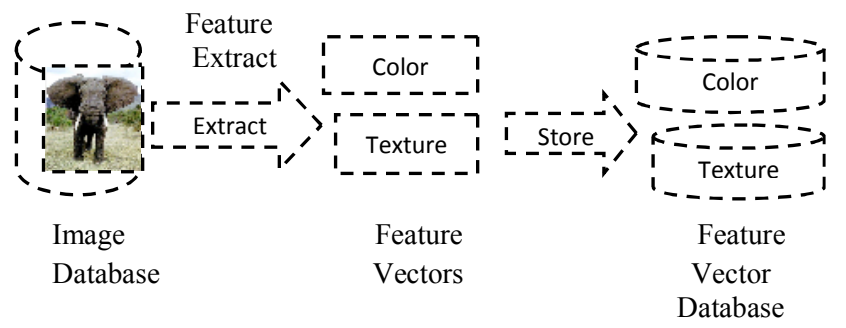

Figure 8 Off Line Process of Feature Extraction

During the online stage, the feature vector of the query image is computed using one selected technique and is compared to all feature vectors in the feature vector database of that technique. Distance metric is used to compute the similarity between feature vectors of the database image. Small distance implies that the corresponding image is similar to the query image and vice versa. Images are retrieved based on increasing distance. The flow of this process is shown in Figure 9.

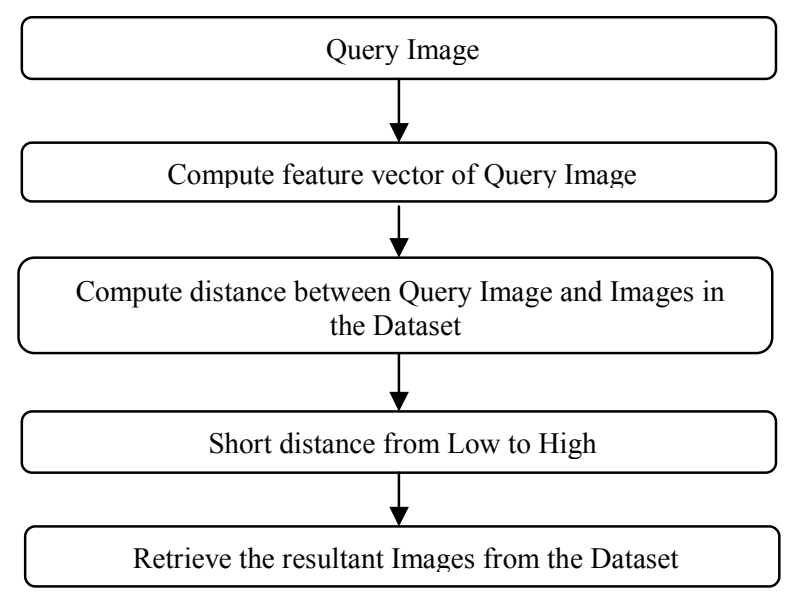

Figure 9. Online Process of Retrieval
By applying the Quantitative Measures and Feature Extraction Techniques like Color Histogram, Gabor and Wavelet Transform with three different Distance Metrics Measures like Euclidean Distance, Chi-Square Distance, and Weighted Euclidean Distance are given in equation 7, 8,9. to all 800 images in the dataset. The quantitative measures are calculating the Sensitivity and Specificity for the image. The sensitivity SE (or True Positive rate) as

$$
S E=\frac{T P}{T P+F N}----(10)
$$

And the specificity SP (or True Negative rate) as

$$
S P=\frac{T N}{T N+F P}----
$$

Where,

TP - True Positive is equivalent with hit data.

$\mathrm{TN}$ - True Negative is equivalent with correct rejection data.

FP- False Positive is equivalent with false data.

FN - False Negative is equivalent with miss data.

From the quantitative measures the Gabor Transform gives the best result with more execution time, compared it with other technique. From the equation 10, the comparative chart of quantitative measure based upon sensitivity is shown in Figure 10

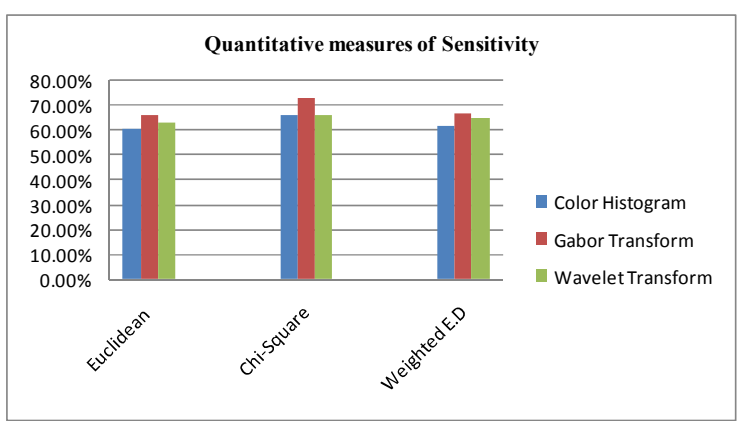

Figure 10.Comparision of quantitative measures based upon Sensitivity.

From the equation 11 the comparative chart based upon Specificity is in Figure 11.

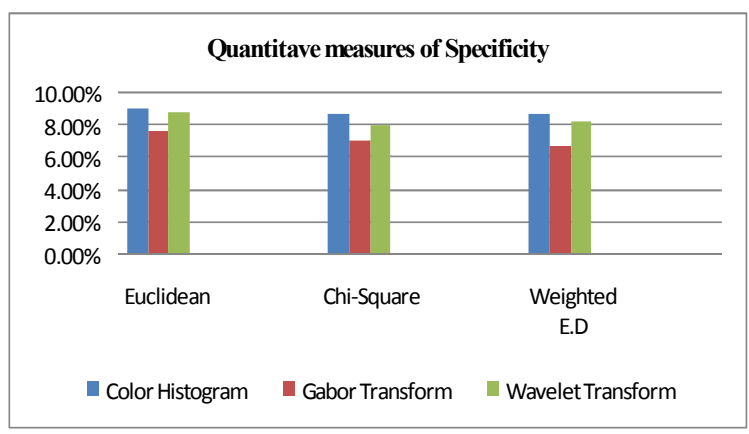

Figure 11. Comparison of Quantitative measures based upon Specificity 
From this analysis, the Gabor Transform with Chi-Square Distance Metric Measure gives the better performance. The average execution time taken by three techniques with three different distances metric measures are given in the comparison chart shown in Figure 12. Wavelet transform retrieve the image at very fast compared it with other techniques; but gives the poor performance, similarly color histogram also. But Gabor transform takes much time but gives the best result.

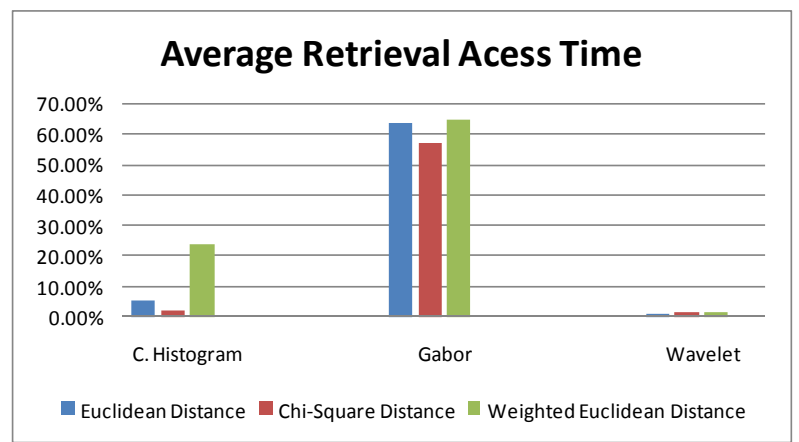

Figure 12. Retrieval access time with all distance measures

The comparative chart table of sensitivity and specificity in percentage is in Table 1.

\section{TABLE 1 COMPARATIVE CHART OF SENSITIVITY \&} SPECIFICITY

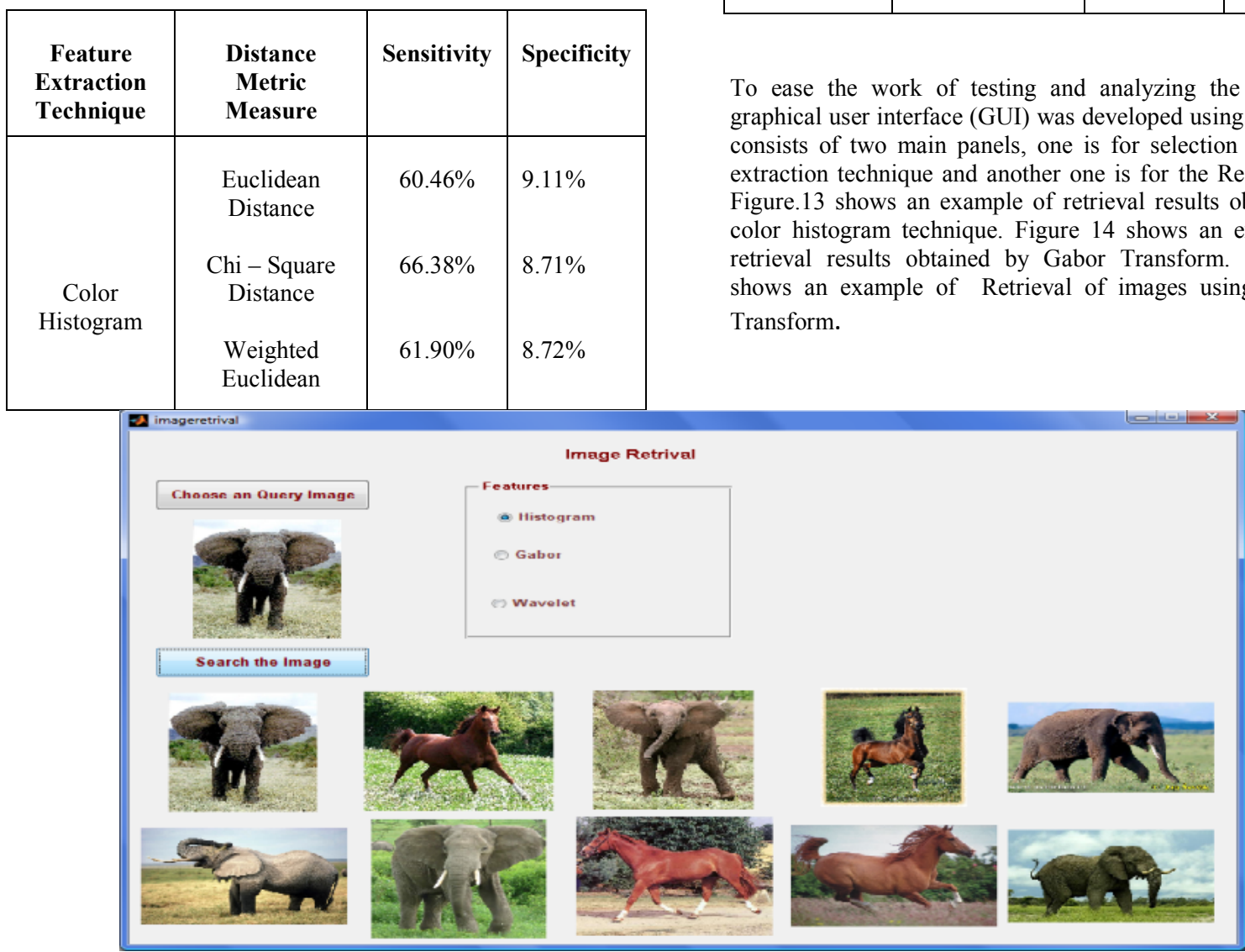

Figure 13. Example of Retrieval of images using Color Histogram 


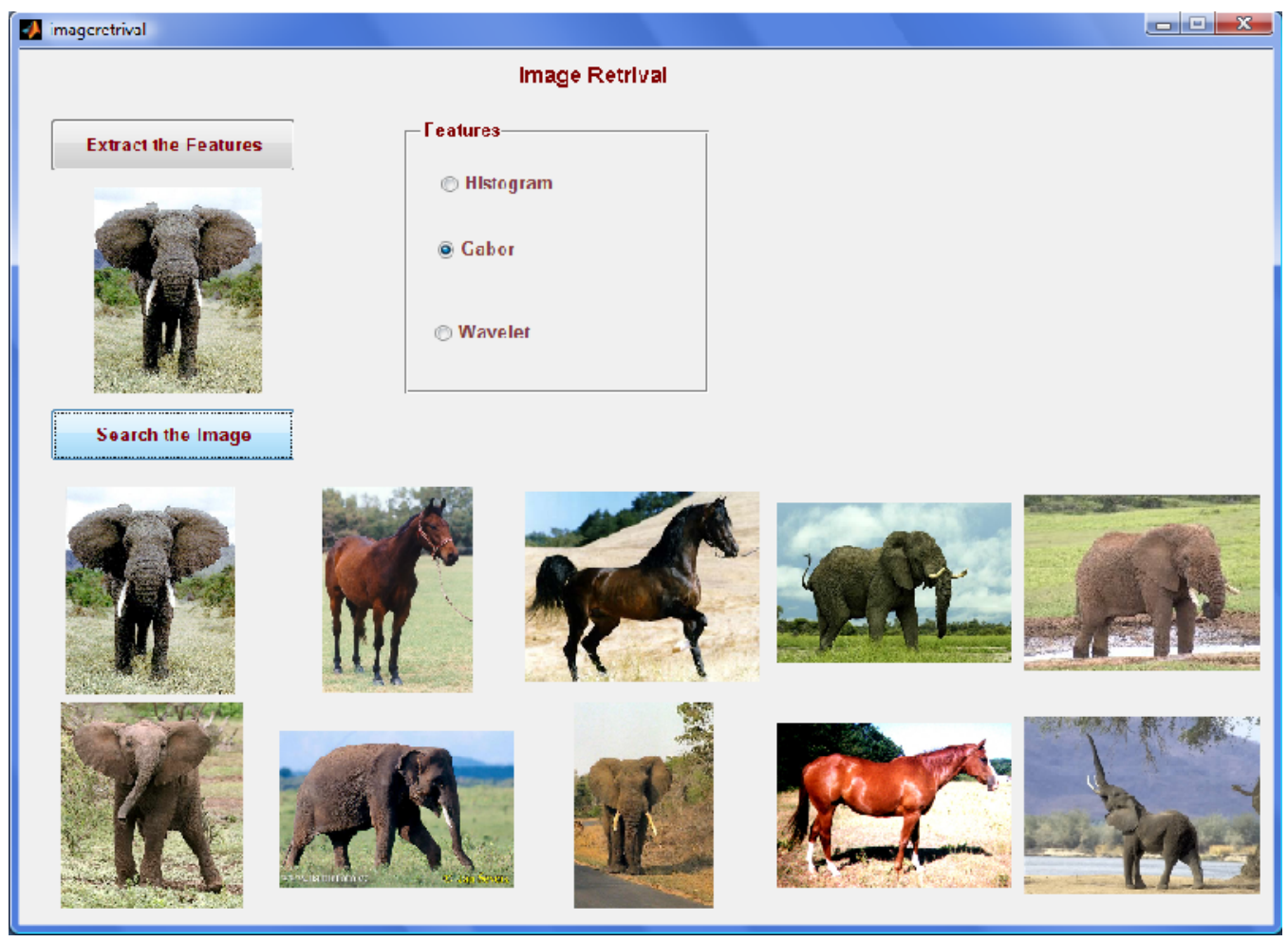

Figure 14 Example of Retrieval of images using Gabor Transform

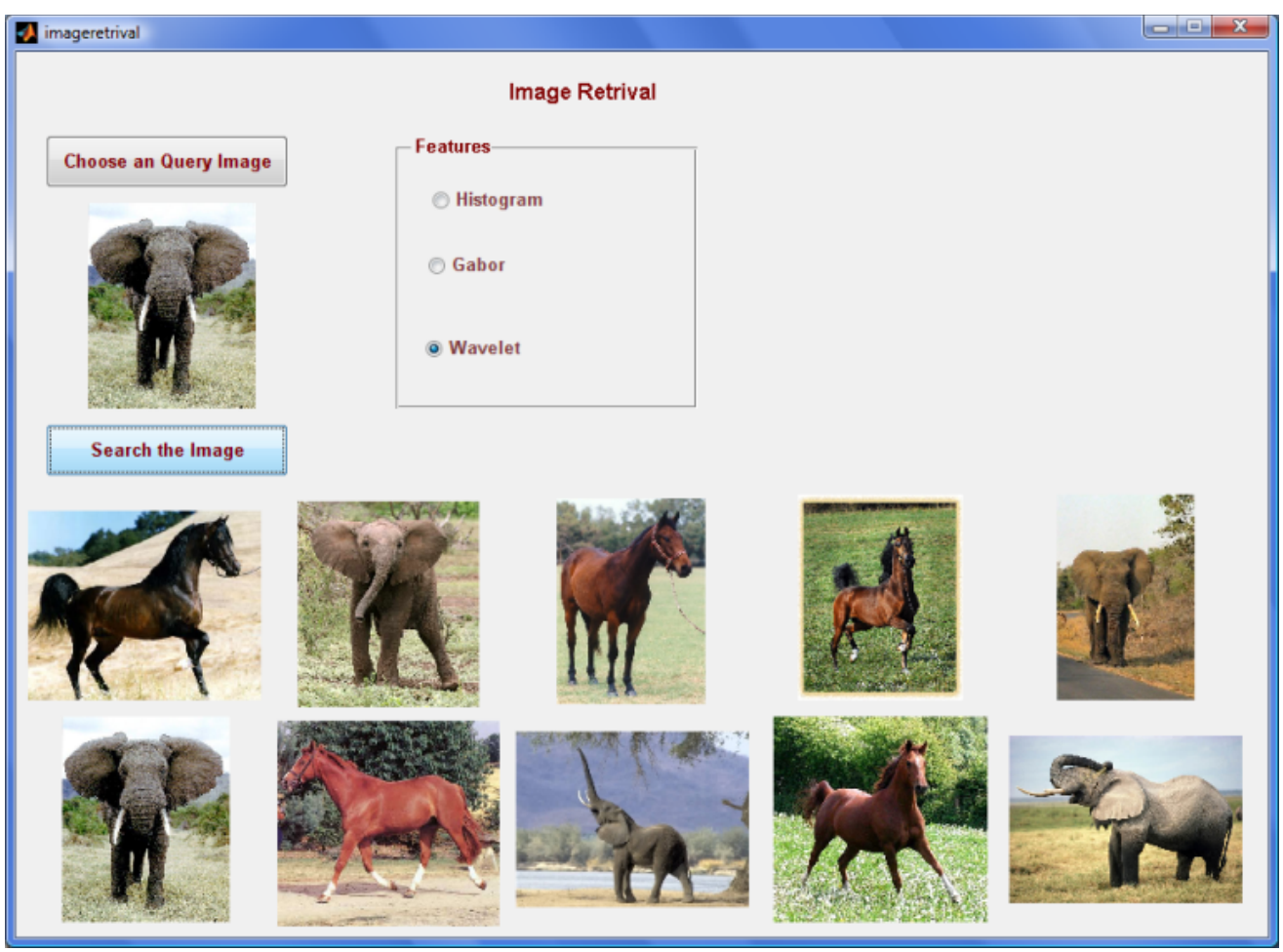

Figure 15. Example of Retrieval of images using Wavelet Transform 


\section{CONCLUSIONS}

In this paper, a comparative study has been carried out on three feature extraction techniques and extracts the feature of texture, Color of color image with three different distance metrics measures are taken for retrieving the similar images from the data set. From this experiment more images are retrieved by using Gabor than the other two feature extraction techniques like Color Histogram, Wavelet Transform. The future work is, doing the feature extraction of the image with another technique and with different distance measure to give the better result. Therefore to provide better performance of retrieving more images are needed on the basis of these techniques.

\section{ACKNOWLEDGEMENT}

The authors would like to thank the anonymous reviewers for their thorough reviews, and constructive suggestions which significantly enhance the presentation of the paper.

\section{REFERENCES}

[1] N. S. Chang and K.S.Fu, 1980, "Image query by Pictorial example," IEEE Trans Software Engineering.
[2] Pengyu Liu, Kebin Jia, Zhuozheng Wang, ZhuoyiLv 2007, "A New and Effective Image Retrieval Method Based on Combined Features “. IEEE.

[3] Y. Rui and T.S. Huang . 1999, 10, pp. 39-62. "Image Retrieval: current techniques, promising directions, and Open issues, Visual Commun, Image Representation.

[4] A.W.M. Smeulders, et al., 2000 "Content-based image retrieval at the end of the early years", IEEE Trans. Pattern Anal. Mach. Intel, 22, pp. 1349-1379.

[5] Stephane Mallet, 1996, April,"Wavelets for a Vision. Proceeding to the IEEE, Vol. 84:604-685.

[6] Jan de leeuw, sandra pruzansky, December, 1978 A new computational method to fit the weighted Euclid Distance Model, Psychometrika--vol. 43, no. 4.

[7] Tomasz Andrysiak, Michał Chora', 2005 "Image Retrieval Based On Hierarchical Gabor Filters", Int. J. Appl. Math. Comput. sci., , Vol. 15, No. 4, 471-480

[8] Wan Siti Halimatul Munirah Wan Ahmad and Mohammad Faizal Ahmad Fauzi, 2008," Comparison of Different Feature Extraction Techniques in ContentBased Image Retrieval for CT Brain images", IEEE,

[9] Wei-Ta Chen, Wei-Chuan Liu, and Ming-Syan Chen, August 2010. "Adaptive Color Feature Extraction Based on Image Color Distributions", IEEE Transactions on Image Processing, Vol. 19, No. 8. 